\section{Changes of circulating blood volume in coronary arterial graft surgery with and without cardiopulmonary bypass}

To the Editor:

We evaluated the circulating blood volume (BV) of patients who underwent cardiac surgery with or without cardiopulmonary bypass $(\mathrm{CPB})$ by using the new pulse dye-densitometry (PDD) method, ${ }^{1-3}$ to measure whether there are differences in circulating BV changes during and after surgery.

After approval by our Institutional Ethics Committee and informed consent, anesthesia for patients with $(n=12)$ and without $(n=12)$ CBP was induced by midazolam, fentanyl, and vecuronium. Anesthesia was then maintained with midazolam and fentanyl, and/or sevoflurane. Before (preoperative) and after surgery (postoperative), including arrival in the intensive care unit, at 9:00 a.m. (POD 1) the morning after surgery, and two days afterwards at 9:00 a.m. (POD 2), BV was measured by the usual PDD methods using a DDG analyzer (Nihon Kohden Co., Tokyo, Japan). No significant differences between the $\mathrm{CPB}$ vs the non-CPB group were observed in age, weight, height, duration of operation and anesthesia. The changes of $\mathrm{BV}$ and fluid balance values are shown at the four different times measured, in the accompanying Table. BV in the $\mathrm{CPB}$ group decreased postoperatively and during POD 1 , whereas $\mathrm{BV}$ in the non-CPB group did not change. A significant difference in $\mathrm{BV}$ was observed between the $\mathrm{CPB}$ and non-CPB groups at POD 1.

Our results suggest that the $\mathrm{BV}$ of patients undergoing coronary arterial graft surgery with $\mathrm{CPB}$ decreases after surgery and gradually recovers to its preoperative level, whereas the $\mathrm{BV}$ in patients undergoing surgery without $\mathrm{CPB}$ does not change postoperatively.

Mitsuaki Yamazaki MD

Kiyoshi Shakunaga MD

Koki Hirota MD

Toyama, Japan

\section{References}

1 Iijima T, Aoyagi T, Iwao $\Upsilon$, et al. Cardiac output and circulating blood volume analysis by pulse dye- densitometry. J Clin Monit 1997; 13: 81-9.

2 Haruna M, Kumon $K$, rabagi $N$, et al. Blood volume measurement at bedside using ICG pulse spectrophometry. Anesthesiology 1998; 89: 1322-8.

3 Iijima T, Iwao $\Upsilon$, Sankawa $H$. Circulating blood volume measured by pulse dye-densitometry. Comparison with ${ }^{131}$ I-HSA analysis. Anesthesiology 1998; 89 1329-35.

\section{Identification of the thoracic epidural space by the running infusion drip technique}

\section{To the Editor:}

Location of the thoracic epidural space between T3 and T7 is difficult because of the extreme caudad angulation of the long posterior spinous processes at these levels. Also, above L2, the epidural space gradually becomes smaller because of the size of the spinal cord.

The loss-of-resistance technique is the most frequently used method for locating the epidural space. However, because of the difficulty of identification of

TABLE Changes of BV and in-out balance values

\begin{tabular}{|c|c|c|c|c|c|}
\hline & Preoperative & Postoperative & & $P O D 1$ & POD 2 \\
\hline \multicolumn{6}{|l|}{$B V(L)$} \\
\hline CPB & $5.11 \pm 1.64$ & $3.73 \pm 1.02 \mathrm{a}$ & & $3.81 \pm 0.49 \mathrm{a}$ & $4.73 \pm 1.13$ \\
\hline \multirow[t]{2}{*}{ non-CPB } & $5.29 \pm 0.97$ & $4.23 \pm 1.16$ & & $5.21 \pm 1.21^{*}$ & $5.44 \pm 1.24$ \\
\hline & & Prepost & Post-POD 1 & \multicolumn{2}{|r|}{ POD1-POD 2} \\
\hline \multicolumn{6}{|c|}{ Fluid balance ( $m L)$} \\
\hline CPB & & $4890 \pm 1572$ & $478 \pm 526$ & \multicolumn{2}{|r|}{$290 \pm 694$} \\
\hline non-CPB & & $3188 \pm 773^{*}$ & $-59 \pm 754$ & \multicolumn{2}{|r|}{$-789 \pm 607^{*}$} \\
\hline
\end{tabular}

$\mathrm{BV}=$ circulating blood volume, fluid balance=fluid transfusion volume-blood loss-urine output; Prepost=fluid balance during anesthesia; Post-POD $1=$ fluid balance from postoperative to POD $1 ;$ POD $1-P O D 2=$ in-out balance from POD 1 to POD $2 ; \mathrm{CPB}=$ cardiopulmonary bypass.

a: different from preoperative (ANOVA, $P<0.05$ )

${ }^{*}$ Different from CPB group (non-paired t test, $P<0.05$ ).

Results are mean \pm SD 


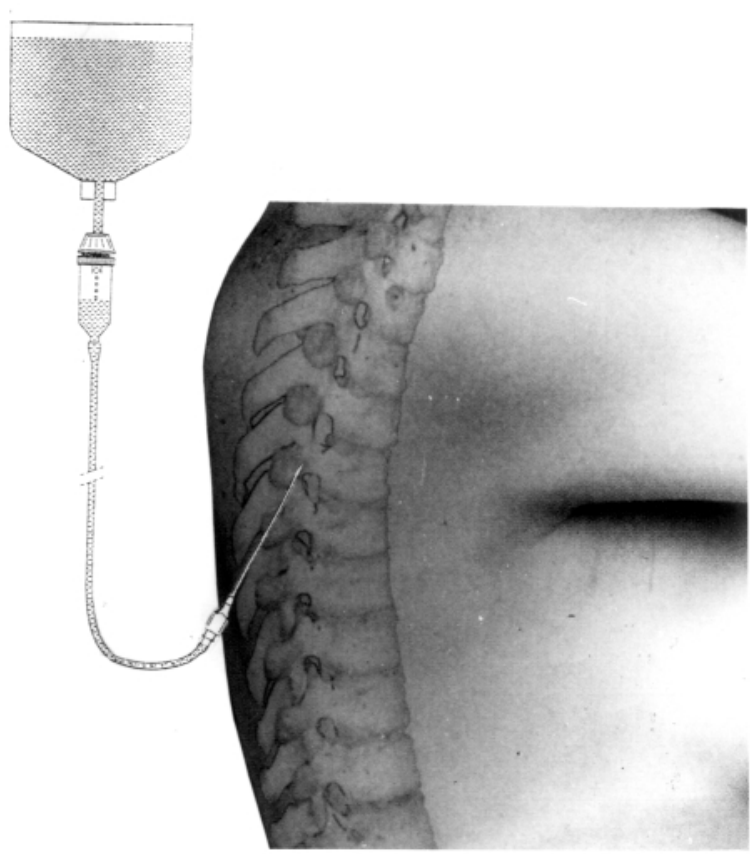

FIGURE A saline infusion set is positioned approximately one metre above the site of proposed epidural anesthesia and connected to the hub of the epidural needle. As soon as the needle pierces the ligamentum flavum, and enters the peridural space, the infusion starts to drip due to the hydrostatic pressure difference between the infusion and the peridural space.

the epidural space in the thoracic region as well as the possibility of inadvertent dural puncture resulting in intrathecal administration of the local anesthetic and/or spinal cord injury, an objective technique for identification of the thoracic epidural space must be considered as an alternative for the subjective loss-ofresistance technique.

Thirty years ago, I reported the use of the running infusion technique for identification of the lumbar epidural space. ${ }^{1}$ The technique has also proved to be very useful for identifying the thoracic epidural space. Patients are positioned in the sitting position and, after scrubbing and draping the skin, a Tuohy epidural needle is inserted in the midlle line at the desired thoracic level, with an upward angulation of 45-60 angle to the skin. The needle is advanced through the skin, subcutaneous tissue, supraspinous ligament and interspinous ligament. The stylet is then removed, and the sterile end of a saline infusion drip tubing is carefully connected to the hub of the needle. The drip is then left open. The infusion sometimes drips slowly when the tip of the needle is in the loose interspinous ligament. However, when the needle engages the ligamentum flavum, marked resistance is felt and the infusion always stops running. The needle is then advanced very slowly through the ligamentum flavum. As soon as the bevel of the needle enters the epidural space, the infusion starts to flow, thus identifying the epidural space (Figure). The rate of the drip varies from patient to patient, due to possible variations in the epidural pressure. Entry into the space is usually associated with a "give way" feeling and advancement of the needle is stopped immediately. The infusion is then detached from the needle. If no cerebrospinal fluid drips from the needle, injection of $3 \mathrm{~mL}$ of lidocaine plus epinephrine $1: 200,000$ is used as a test dose. The injection without resistance, as well as the absence of tachycardia or spinal anesthesia confirm proper epidural localization.

\section{Anis Baraka MD FRCA}

Beirut, Lebanon

\section{Reference}

1 Baraka A. Identification of the peridural space by a running infusion drip. British Journal of Anaesthesia 1972; 44: 122 .

\section{Gauging the depth of venipuncture dur- ing internal jugular vein cannulation}

To the Editor:

Right internal jugular vein (IJV) is the preferred site for central venous cannulation. During the procedure there are chances of complications like arterial puncture, hematoma formation, pneumothorax, thoracic duct injury, arteiovenous fistula, and brachial plexus injury due to the inability to judge the appropriate length that the large bore needle traverses in order to do the venipuncture. ${ }^{1}$ Complications can be life threatening and may be avoided if the depth from skin to vein is measurable.

In an attempt of safer and accurate cannulation of IJV, we devised a venipuncture and a 24 -gauge guide puncture needle with centimetre-highlighted markings (Figure). The guide puncture determines the direction of IJV and centimetre markings on it give the exact distance at which IJV is hit. Then the venipuncture needle is introduced in the same direction as the guide puncture needle. As the distance from skin entry to the IJV is measured on the guide puncture needle, the venipuncture needle is introduced to the prefixed distance to hit the IJV. 